\title{
Practical prosthod
for the dental team
}

\section{By R.Dubal, ${ }^{\top}$ S.Buth}

\section{Introduction}

Teamwork is an essential aspect of good clinical practice, clinical governance and for ensuring optimal patient care. An effective patient pathway involves interaction with a number of different members of the dental team, all aimed at providing the best possible outcome for the patient. Each member of the team has an influential role to play in making the care pathway as efficient and as smooth as possible, not only for the patient but also for the dental team. Poor teamwork can lead to breakdown in communication and deterioration in working relationships, ultimately leading to sub-standard patient care and a loss of patient trust and confidence.

The General Dental Council (GDC) have stated in their guidance document 'Principles of dental team working' that a good team includes the following components:

1. Good leadership ${ }^{1}$

2. Clear, shared aims, and work together to achieve them ${ }^{1}$

3. Different roles and responsibilities, and understand those roles and responsibilities. ${ }^{1}$

The most recent figures from the GDC report that 40,721 dentists are currently registered to practise in the United Kingdom. ${ }^{2}$ As the workforce increases in size, care teams increase in number and diversify in function. This is reflected in the emergence of dental therapists, orthodontic therapists, clinical dental technicians and oral and maxillofacial clinical scientists. With increasingly more diverse skills available and a multitude of roles to be fulfilled, the pillars of effective communication, teamwork and patient-centred outcomes become even more important. Prosthodontic procedures involve a wide array of individuals with a variety of skills, who come together to ensure high quality rehabilitation for patients.

\section{Prosthodontics}

Prosthodontic procedures are the commonest treatment modality undertaken by dental surgeons. Prosthodontic care includes decision-making, planning and the provision of fixed, removable and implant-retained prostheses. Fixed prostheses include restorations such as crowns, bridges, inlays and onlays. Removable prostheses include acrylic and cobalt chromium dentures. Implant retained prostheses can be fixed or removable including crowns, bridges and dentures.

Twenty-first century prosthodontics has moved a long way from conventional treatments and treatment planning. The emphasis is now very much on the preservation of natural tooth tissue and the use of enamel and dentine bonding wherever possible. The modern prosthodontist is more aware of the implications of failure cycling and is keener to buy biological time.

The Adult Dental Health Survey (2009) revealed that in England, Wales and Northern Ireland, $94 \%$ of the combined population were dentate, meaning that they had one or more natural teeth. ${ }^{3}$ With the current population of the previously mentioned countries estimated to be 57.9 million people, this leaves just under 3.5 million people with no teeth at all. Further to this, $13 \%$ of adults in England had natural teeth with dentures. ${ }^{3}$ The survey also revealed that in England 8\% of dentate adults had one or more carious or unrestorable teeth. ${ }^{3}$ The statistics, when considered in real terms, provide us with the sombre conclusion that the need for care, by means of prevention, restoration and maintenance, is as important now as it has ever been.

The recent revolution in our understanding and provision of conservative and more adhesive clinical prosthodontic treatment has also led to the evolution of roles within the dental team. There has been a change in the way in which we manage dental disease and rehabilitate patients with missing teeth. Each member of the team has an increasingly more valuable part to play with an emphasis on communication, prevention and the patient being at the centre of the care process. This is reflective of the quality of life and 'patientcentred' approach which we should all be embracing today. With a co-operative and focused patient, it is more likely that our direct and indirect restorations will perform better

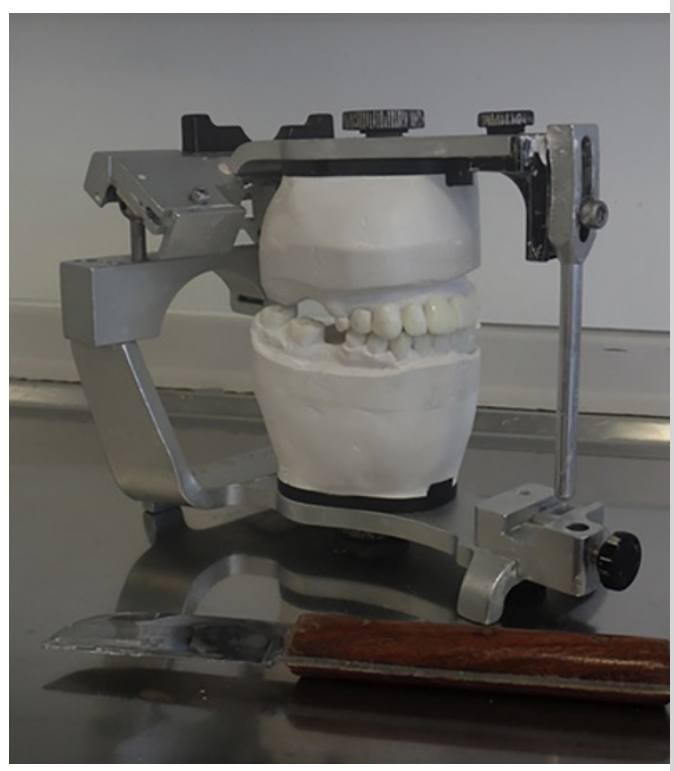

with less associated biological damage at the time of failure.

There has been a move towards the whole dental team being involved in patient care. For most patients, the care pathway begins from the moment they enter the practice reception area, before they even see a dentist.

The receptionist, oral health educator, hygiene and therapy team, dental nurse, dentist and technician, are all responsible in ensuring that the patient is cared for optimally. This is facilitated by good communication and organisation within the team. The roles of each team member are distinct and valuable, and all contribute to the patient's summative experience. In this article we will consider the various roles of each member.

\section{The role of the clinician}

Prosthodontic treatment requires a healthy and stable periodontal foundation. A successful approach and response to oral health promotion requires engagement and involvement between the patient and the dental team. Whilst dentists lead, guide and coordinate clinical pathways, input from all

Raj Dubal is a specialty trainee in restorative dentistry; ${ }^{2}$ Shabana Buth is a dental core trainee year 3 
members of the multidisciplinary team is crucial. Clinicians have the responsibility of ensuring clear management plans and effective utilisation of the multidisciplinary approach to optimise patient outcomes. The team (dentist, therapist, oral health advisor, dental hygienist and dental nurse) must all share a common philosophy towards optimising patient care. Patients with gingival inflammation are rarely good candidates for prosthodontic treatment. Gingival inflammation can give rise to problems with impression taking, the cementation of adhesive restorations such as veneers and the use of composite resin restorations. Many patients believe that the easiest way to solve this problem is to ask for scaling from a hygienist or therapist on a regular basis rather than take responsibility for their own ongoing care. This would be by means of more aggressive daily homecleaning, ensuring that the bristles of their toothbrush and interdental brushes remove debris from the gingival margin and pass subgingivally where inflammation or pocketing may be present. This may mean both pain and gingival bleeding for the patient in the first few weeks of using a more aggressive approach.

The whole team must be united with a common message that patients must take responsibility for their own periodontal health and clean themselves, despite discomfort and bleeding, in order to establish and maintain periodontal health. Although the dentist has the role of leading and coordinating care, they must also help build confidence and empower and encourage all members of the team to promote excellent communication and care when interacting with patients. They will also need to reassure and support team members when patients question key beliefs of the practice, particularly if they are new to them. Utilising the full skillset of all members of the team will promote confidence, reflect team cohesiveness and allow more freedom and autonomy as well as provide a better model of care for patients.

All clinical dental team members should be mindful of monitoring and advising on the causes of dental diseases, which include an increasing frequency of consumption of sugars, oral hygiene advice, a reduction in alcohol consumption and encouraging smoking cessation activities. Where preventative measures fail, ongoing patient maintenance and support should be provided. This ensures that both successes and failures are shared, with the needs of the patient always put first. Such principles can be taught and encouraged through group training, practice meetings to include team-building sessions and access to relevant CPD.

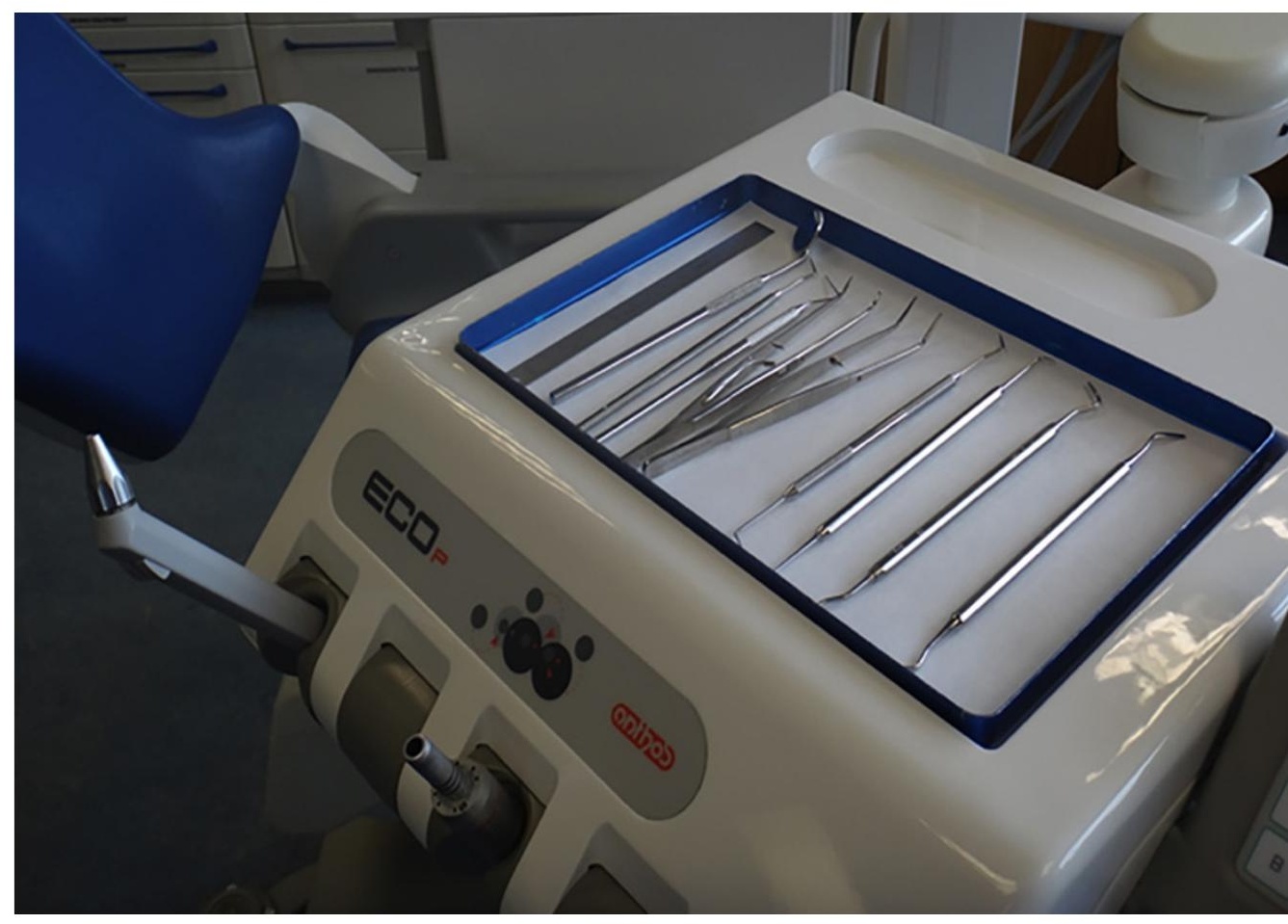

Oral health educators, reception staff and practice managers

The patient journey begins when an appointment is first booked by the receptionist. It is the responsibility of the dentist to assess the patient's suitability for treatment and to gain informed consent. Once a diagnosis is made, a treatment plan can be formulated and treatment options discussed and agreed. It can be very helpful for the oral health educator to contribute to these discussions, particularly when they fully understand the treatment modalities concerned. Sometimes patients are more reluctant to discuss their queries with the dentist, and more comfortable with another member of the team; it is not unusual for a patient to have queries and a discussion with the receptionist first as a result of confusion or misunderstanding. Information leaflets allow the patient to gather information and develop an understanding in their own time and at their own pace. This can help to provide clarity, raise questions and reinforce consent.

Where oral hygiene is inadequate, it may be appropriate to refer the patient to an oral health advisor, dental therapist or hygienist for periodontal treatment and oral hygiene instructions. Ultimately, good oral hygiene and periodontal health is essential for satisfactory prosthodontic care and long term success.

\section{Dental nurses}

The involvement and opinion of the dental nurse can help validate subjective decisions such as choice of tooth shade, or offering an opinion on overall appearance of fixed or removable prosthodontic restorations. It is important that all team members are encouraged to bring their own genuine opinion when giving such advice. In general, two views are better than one, and a more inclusive dimension to patient care is delivered.

Following successful completion of the procedure, dental nurses are fundamental in ensuring that the patient is well, and can provide any post-operative advice, as well as reassurance that may be necessary. They can then direct the patient towards the reception area.

The dental nurse's role also includes adherence to infection control policies, whilst the surgery is cleared, cleaned and disinfected, ready for the next patient. Impressions and any other laboratory work should be disinfected, and packaged according to recommended guidelines prior to dispatch. The nurse is responsible for ensuring the completion of

\section{'The involvement} and opinion of the dental $\underline{\text { nurse can }}$ help validate subjective decisions...' 
appropriate patient identifying labels and disinfection of the laboratory work including the clinician's laboratory instructions. They are also instrumental in ensuring that the correct return dates are noted on the correct laboratory prescription form. There is an intricately efficient inter-play between clinician, nurse, patient, technician and receptionist, and this can be reflective of teamwork at its best. It is clear that when we work together everyone benefits.

\section{The role of dental technicians}

Dental technicians are registered dental professionals ${ }^{4,5}$ who construct devices to a prescription from a dentist or clinical dental technician. ${ }^{4,5}$

They are very important members of the prosthodontic team. Depending on their training and registration they either construct restorations (dental technician) or additionally provide the clinical stages involved in the provision of removable prostheses (clinical dental technician). Dental technicians are involved in the construction of dentures, crowns, bridges and orthodontic appliances aimed at improving the patient's appearance, speech and masticatory function. Dental technicians are divided into four key groups: prosthodontic, conservation, orthodontic and maxillofacial technicians, depending on the type of devices they are trained to fabricate. High standards of care must be shared by all members of the team. Technicians must feel able to inform dentists if they are unhappy with clinical quality issues (eg tooth preparation, denture design, impression quality) and vice versa.

A good relationship between the clinician and dental technician is fundamental in order to achieve prosthodontic success and contributes to optimal treatment outcomes. Poor communication can lead to inadequately constructed or ill-fitting prostheses as well as laboratory work not arriving in time for a patient's appointment. Thus it is highly important, for clinicians to develop a good working relationship with their dental technician colleagues, so that they can freely and clearly communicate the type of appliance desired, material of choice as well as any additional information such as marginal position, type of marginal finish and any specific features pertinent to the case.

\section{Clinical dental technicians}

Clinical dental technicians (CDTs) are registered dental professionals who provide complete dentures directly to patients and other dental devices on prescription from a dentist. ${ }^{5}$ They are also qualified as dental technicians. The key difference is that they are trained to undertake a detailed dental history and relevant medical history ${ }^{5}$ and perform any technical and clinical procedures related to providing removable dental appliances. ${ }^{5}$ They are also able to prescribe, provide and fit removable prostheses to patients directly.

The number of qualified CDTs emerging is increasing and some may argue that they are now a separate entity to clinicians when it comes to prescribing, fabricating and fitting certain types of removable prostheses. However, it is also important to note that although CDTs may be able to set up their own independent practice, they must have adequate protocols in place to enable appropriate patient referrals when faced with a patient whose needs are out of their scope of practice. ${ }^{4,5}$ This is critical in order to ensure an efficient and successful patient care pathway, which is also robust and comprehensive.

Many technicians have valuable insight and experience regarding recording tooth shade for a prosthesis, and the management of challenging cases. In some cases it is valuable to call the lab or even visit them so that the case can be discussed and a combined plan of action be established. Careful planning and the combination of skill sets can ensure optimal treatment outcomes.

Overall, a sound working relationship and clear communication between the prescribing clinician and the dental or clinical dental technician is of paramount importance to ensure both accurate and efficient patient treatment with successful outcomes. This is an integral part of the patient care pathway.

\section{Summary}

There has been a paradigm shift towards a patient-centred approach to delivering high quality prosthodontic care. As a function of this, the patient-care pathway should be supportive, engaging and informed at all points of the journey. The calming effect of a considerate receptionist is often the first and last point of contact for patients, and the psychosocial and emotional dimension of the care experience is what patients often judge us on.

As the roles and responsibilities of team members continue to evolve, the overlap of each team member's duties become wider, and this should allow us to create a more comprehensive care network. Irrespective of individual roles, there should be a focus on empathetic and effective communication with the patient, which should be honest, comprehensive and comprehendible. All team members should be knowledgeable and be able to answer or redirect common questions asked by patients.

In consideration of the GDC and Care
Quality Commission aspirations to set and ensure well outlined standards for the profession, there is a shared and implicit duty on the part of all members of the team to ensure that these are met and that relevant policies and best practice guidelines are adhered to. Now is an exciting time for evolution of the dental team, and never has there been greater access to opportunities for professional growth and development. Continuing training and education for all members of the team and regular audit will help to ensure that we are all doing what we are supposed to be doing, and this will equip us to improve our services further.

In the twenty-first century, with a greater number of more complex prosthodontics treatments available, it is increasingly important that treatment planning, explanations of treatment options and the provision of successful prosthodontic care are provided by a high quality dental team. As a result, we must work together with a common goal and ideologies, which place our patients at the centre of our focus. As they say, there is no I in team...

1. General Dental Council. Principles of dental team working. Available from: http://www. gdc-uk.org/dentalprofessionals/standards/ documents/principlesofdentalteamworking br0591_1.pdf (accessed 1 November 2015).

2. General Dental Council. Facts and Figures : Registrant Reports. Available from: https:// www.gdc-uk.org/Newsandpublications/ factsandfigures/Documents/Facts\%20and\%20 Figures\%20from\%20the\%20GDC\%20 register\%20October\%202015.pdf (accessed 1 November 2015).

3. Health and Social Care Information Centre. Executive Summary: Adult Dental Health Survey 2009. Available from: http://www. hscic.gov.uk/catalogue/PUB01086/adul-dentheal-surv-summ-them-the1-2009-rep3.pdf (accessed 1 November 2015).

4. General Dental Council. Scope of practice. Available from: https://www.gdc-uk. org/Newsandpublications/Publications/ Publications/Scope\%20of\%20Practice $\% 20$ September\%202013.pdf. (accessed 1 November 2015).

5. NHS Education for Scotland. A career in clinical dental technology. Available from: http://www.nes.scot.nhs.uk/media/2534970/ nesd0155_clinicaldentaltech.pdf (accessed 1 November 2015). 